\title{
Magnetic susceptibility of alkali-TCNQ salts and extended Hubbard models with bond order and charge density wave phases
}

\author{
Manoranjan Kumar, Benjamin J. Topham, Rui Hui Yu, Quoc Binh Dang Ha*, Zoltán G. Soos ${ }^{\dagger}$ \\ Department of Chemistry, \\ Princeton University, Princeton NJ 08544
}

(Dated: November 11, 2018)

\begin{abstract}
The molar spin susceptibilities $\chi(T)$ of Na-TCNQ, K-TCNQ and Rb-TCNQ(II) are fit quantitatively to $450 \mathrm{~K}$ in terms of half-filled bands of three one-dimensional Hubbard models with extended interactions using exact results for finite systems. All three models have bond order wave (BOW) and charge density wave (CDW) phases with boundary $V=V_{c}(U)$ for nearest-neighbor interaction $V$ and on-site repulsion $U$. At high $T$, all three salts have regular stacks of $\mathrm{TCNQ}^{-}$anion radicals. The $\chi(T)$ fits place $\mathrm{Na}$ and $\mathrm{K}$ in the $\mathrm{CDW}$ phase and $\mathrm{Rb}(\mathrm{II})$ in the BOW phase with $V \approx V_{c}$. The $\mathrm{Na}$ and $\mathrm{K}$ salts have dimerized stacks at $T<T_{d}$ while $\mathrm{Rb}(\mathrm{II})$ has regular stacks at $100 \mathrm{~K}$. The $\chi(T)$ analysis extends to dimerized stacks and to dimerization fluctuations in $\mathrm{Rb}(\mathrm{II})$. The three models yield consistent values of $U, V$ and transfer integrals $t$ for closely related TCNQ ${ }^{-}$stacks. Model parameters based on $\chi(T)$ are smaller than those from optical data that in turn are considerably reduced by electronic polarization from quantum chemical calculation of $U, V$ and $t$ on adjacent $\mathrm{TCNQ}^{-}$ions. The $\chi(T)$ analysis shows that fully relaxed states have reduced model parameters compared to optical or vibration spectra of dimerized or regular $\mathrm{TCNQ}^{-}$stacks.
\end{abstract}

\section{INTRODUCTION}

The strong $\pi$-acceptor $\mathrm{A}=$ TCNQ (tetracyanoquinodimethane) forms an extensive series of ion-radical salts [1-3] with closed-shell inorganic ions as well as charge-transfer (CT) complexes with $\pi$-donors such as $\mathrm{D}=\mathrm{TTF}$ (tetrathiafulvalene). The high conductivity and phase transitions of TTF-TCNQ on cooling were thoroughly investigated as an important step towards the realization of organic superconductivity [4]. TCNQ salts crystallize in face-to-face stacks that immediately rationalize their quasi-one-dimensional (1D) electronic structure. Endres has reviewed the many structural motifs of 1D stacks [5]. We consider in this paper the magnetic properties of "simple" 1:1 alkali-TCNQ salts with half-filled stacks of $\mathrm{A}^{-}$radical ions. Complex salts with stoichiometry such as 1:2 or 2:3 have less than half-filled stacks; they are semiconductors with higher conductivity than simple salts. Hubbard and related models are the standard approach to TCNQ salts or CT complexes [1-4]. Each molecule in a stack is a site with a single frontier orbital, the lowest unoccupied orbital of A or the highest occupied orbital of D.

Heisenberg spin chains were initially applied to the magnetic properties of TCNQ salts [6], especially to dimerized stacks whose elementary excitations are triplet spin excitons. Subsequently, Hubbard models [1 4] opened the way to discuss optical and electrical as well as magnetic properties. Limited understanding of

* Present address: University College Dublin, Dublin Ireland 01716 7777

${ }^{\dagger}$ Electronic mail:soos@princeton.edu
1D models hampered early treatments. Theoretical and numerical advances now make it possible to treat the spin susceptibility of 1D models almost quantitatively. Alkali-TCNQ salts offer the possibility of joint modeling of magnetic, optical and vibrational properties. An interesting consequence reported below is that different model parameters are needed for magnetic and optical properties.

There is considerable literature on K-TCNQ or NaTCNQ, recently in connection with photo-induced phase transitions [7, 8]. They form [5] regular stacks with $\mathrm{A}^{-}$ at inversion centers at high $T$, dimerized stacks with two $\mathrm{A}^{-}$per repeat unit at low $T$. The transitions are 9] at $T_{d}=348 \mathrm{~K}$ and $395 \mathrm{~K}$, respectively, for $\mathrm{Na}$ and K-TCNQ. Torrance [10] and others [11] sought to model $T_{d}$ as a spin-Peierls transition, as discussed in the review of Bray et al. [12] who noted that such high $T_{d}$ requires unacceptably large exchange constants. The transitions have some $3 \mathrm{D}$ character since the cations also dimerize $[13,14]$. We model the molar spin susceptibility $\chi(T)$ of the $\mathrm{Na}$ and $\mathrm{K}$ salts at $T>T_{d}$ using regular stacks. We also consider $\chi(T)$ of dimerized stacks for $T<T_{d}$ without, however, treating the transition. There are two Rb salts: Rb-TCNQ(I) is strongly dimerized [15] at $300 \mathrm{~K}$ while Rb-TCNQ(II) has regular stacks [16, 17] with $A^{-}$at inversion centers at both 100 and $295 \mathrm{~K}$. The recent $100 \mathrm{~K}$ structure [17] rules out a dimerization transition around $220 \mathrm{~K}$ that was inferred from magnetic susceptibility [18] and infrared [19] data. We reinterpret these observations. Regular stacks make Rb-TCNQ(II) the best target for modeling $\chi(T)$.

Fig. 1 shows the molar spin susceptibilities of $\mathrm{Na}, \mathrm{K}$ and $\mathrm{Rb}-\mathrm{TCNQ}$ (II). The $\mathrm{K}$ and $\mathrm{Na}$ data are integrated electron spin resonance (esr) of Vegter and Kommandeur 
[18], who identified the transitions. Dimerization opens a magnetic gap $E_{m}>0$ and rationalizes reduced $\chi(T)$ that vanishes at $T=0$, whether or not $\chi(T)$ can be modeled. Crystal data [13, 14] at $T>T_{d}$ indicate eclipsed (ring over ring, Fig. 1) stacks with $\mathrm{TCNQ}^{-}$ at inversion centers and interplanar separation $\mathrm{R}(\mathrm{Na})$ $=3.385 \AA$ at $353 \mathrm{~K}, \mathrm{R}(\mathrm{K})=3.479 \AA$ at $413 \mathrm{~K}$. The solid line for $\mathrm{Rb}(\mathrm{II})$ is esr intensity [18]. The dotted line is static susceptibility [17] corrected for diamagnetism. The measurements agree at $300 \mathrm{~K}$ and both have a knee around $220 \mathrm{~K}$, less prominently in static susceptibility. The structure has slipped stacks [17] (ring over external bond, Fig. 1) of $\mathrm{TCNQ}^{-}$at inversion centers with $\mathrm{R}$ $=3.174$ and $3.241 \AA$ at 100 and $295 \mathrm{~K}$, respectively. These regular stacks clearly have large $E_{m}$. They are not compatible with finite $\chi(0)$ at $T=0$ and $E_{m}=0$ in regular Heisenberg [20] or Hubbard [21] chains.

The 1D extended Hubbard model [22] (EHM, Eq. 1 below) has nearest-neighbor interaction $V$ in addition to onsite repulsion $U>0$. Increasing $V$ in a half-filled EHM induces a transition to a charge density wave (CDW) phase 22]. The CDW boundary is at $V_{c}(t=0)=U / \alpha_{M}$ in the atomic limit of $U>>t$, where $t$ is electron transfer between adjacent sites and $\alpha_{M}=2$ for the EHM is the Madelung constant of the lattice. The CDW transition is closely related to the neutral-ionic transition of CT salts from largely neutral DADA stacks to largely ionic $\mathrm{D}^{+} \mathrm{A}^{-} \mathrm{D}^{+} \mathrm{A}^{-}$stacks 23 25]. In either case, the ground state (gs) undergoes a first-order quantum transition at small $t / U$ or a continuous transition when $t / U$ exceeds a critical value, or when $U<U^{*}$. Nakamura [26] recognized that the EHM with $U<U^{*}$ has a narrow bond order wave (BOW) phase between the CDW phase at $V_{c}(U)>U / 2$ and the spin-fluid phase with $E_{m}=0$ at $V_{s}(U)<U / 2$. The BOW phase has finite $E_{m}$ in a regular stack and broken inversion symmetry $\mathrm{C}_{\mathrm{i}}$ at sites. Subsequent studies [27-29] confirmed the BOW phase of the EHM and sought accurate values of $V_{s}(U), V_{c}(U)$ and $U^{*}$. We recently characterized the BOW phase of the EHM and related broken $C_{i}$ symmetry to electronic solitons [30]. Finite $E_{m}$ in regular stacks is an attractive way to rationalize $\chi(T)$ in Fig. 1 and we have proposed that Rb-TCNQ(II) is a BOW phase system [17, 31].

In this paper, we model the spin susceptibility of $\mathrm{Na}$, $\mathrm{K}$ and $\mathrm{Rb}-\mathrm{TCNQ}$ (II) quantitatively with the EHM and related models with more realistic Coulomb interactions. We find the $\mathrm{Na}$ and $\mathrm{K}$ salts to be in the CDW phase with $V$ slightly greater than $V_{c}(U)$ and the $\mathrm{Rb}(\mathrm{II})$ salt to be just on the BOW side of $V_{c}(U)$. Modeling $\chi(T)$ is both challenging and decisive for several reasons. First, the full electronic spectrum is required, not just the ground state. Second, comparison with experiment is absolute since the magnitude of $\chi(T)$ follows without scaling in $\pi$-radicals with weak spin-orbit coupling. Third, all three salts have $1 \mathrm{D}$ stacks of $\mathrm{A}^{-}=\mathrm{TCNQ}^{-}$with similar $U$ and other parameters on physical grounds. To the best of our knowledge, Hubbard models have

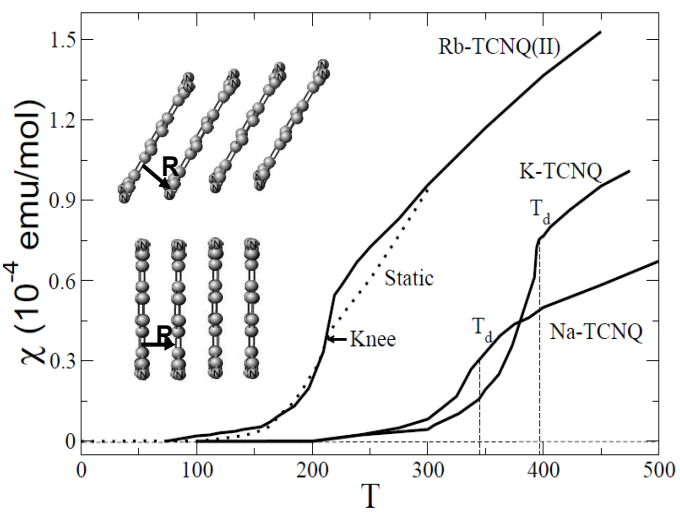

FIG. 1: Solid lines: molar magnetic susceptibility of alkaliTCNQ salts based on electron spin resonance intensity from ref. [18]; dotted line, static susceptibility from ref. [17]. The $\mathrm{Na}$ and $\mathrm{K}$ salts dimerize at $T_{d}$ and have regular faceto-face stacks for $T>T_{d}$ with separation $\mathrm{R}$ between molecular planes. The $\mathrm{Rb}$ salt has regular ring-over-external-bond stacks down to $100 \mathrm{~K}$ and a knee around $220 \mathrm{~K}$.

not been applied quantitatively to both magnetic and optical/vibronic properties of the same system. 1:1 alkali-TCNQ salts provide such an opportunity.

The paper is organized as follows. We present in Section II the spin susceptibility of Hubbard-type models near the boundary $V_{c}(U)$ between the BOW and CDW phases. The magnetic gap $E_{m}(V)$ to the lowest triplet state increases rapidly at $V \approx V_{c}$. In Section III we model the $\chi(T)$ data in Fig. 1 with similar parameters for $\mathrm{TCNQ}^{-}$stacks in related but not identical crystals. We compute model parameters in Section IV for individual $\mathrm{TCNQ}^{-}$or for adjacent $\mathrm{TCNQ}^{-}$. These parameters are reduced substantially in crystals, more so for magnetic than for optical or vibrational properties. The Discussion briefly addresses the parameters of Hückel, Hubbard or other semiempirical models.

\section{BOW/CDW BOUNDARY}

We consider a half-filled extended Hubbard model 22] (EHM) in 1D and extend it to second-neighbor interac- 
tions $V_{2}=\gamma V$. The EEHM with $\gamma>0$ is

$$
\begin{aligned}
H(\gamma) & =\sum_{p=1, \sigma}^{N}-t\left(a_{p, \sigma}^{\dagger} a_{p+1, \sigma}+h . c\right) \\
& +\sum_{p=1}^{N} U n_{p}\left(n_{p}-1\right) / 2 \\
& +\sum_{p=1}^{N} V n_{p}\left(n_{p+1}+\gamma n_{p+2}\right)
\end{aligned}
$$

The first term describes electron transfer between adjacent sites with retention of spin $\sigma$. Regular stacks in this Section have equal $t$ s taken as $t=1$. The number operator is $n_{p}$. The last two terms are on-site repulsion $U>0$, nearest-neighbor interaction $V$ and secondneighbor interaction $\gamma V$. The spin fluid phase with $n_{p}=1$ at all sites is the gs for small $V$ while the charge density wave (CDW) with two electrons per site on one sublattice is the gs for large $V$. The CDW boundary is $V_{c}(t=0)=U / \alpha_{M}(\gamma)$, where $\alpha_{M}(\gamma)=2(1-\gamma)$ is the 1D Madelung constant. As recognized from the beginning [6, 32], electrostatic interactions are 3D and ion-radical organic salts have $\alpha_{M} \approx 1.5$. Point charges in $1 \mathrm{D}$ lead to $\alpha_{\mathrm{M}}=2 \ln 2$. Physical considerations set $\alpha_{M}=1.5$ rather than $\alpha_{M}(E H M)=2$.

Finite $t$ in a regular stack leads to a narrow BOW phase [26] between $V_{s}<U / \alpha_{M}$ and $V_{c}>U / \alpha_{M}$ for $U<U^{*}$, with[29] $U^{*} \approx 7 t$ for the EHM. Smaller $\alpha_{M}$ gives a less cooperative CDW transition and extends the BOW phase to higher $U^{*}$. The point charge model (PCM) with long-range Coulomb interactions $V_{n}=V / n$ in Eq. [1 has [33] $U^{*}(\mathrm{PCM}) \approx 10 t$. By the same analysis, we estimate that the EEHM with $\gamma=0.2$ in Eq. 1 has $U^{*} \approx 9 t$. Quantum chemical evaluation [34] of $U$ and $V$ places alkali-TCNQ salts at the CDW boundary and imposes the constraint $V \approx V_{c}$ in Eq. 1 .

The symmetry properties of $H(\gamma)$ are the same for spin-independent interactions. Total spin $S$ is conserved and $E_{m}$ is from the singlet gs to the lowest triplet state. The half-filled band has electron-hole symmetry $J= \pm 1$. We define $E_{J}$ as the excitation energy to the lowest singlet with opposite $J$ from the gs. A regular stack has inversion symmetry $C_{i}$ at sites that we label as $\sigma= \pm 1$ and define $E_{\sigma}$ as excitation to lowest singlet with opposite $\sigma$ from the gs. The energy thresholds $E_{m}$, $E_{J}$ and $E_{\sigma}$ of extended stacks are not known exactly for $V>0$ in Eq. 1 .

We consider $V \approx V_{c}(N)$ and use valence bond methods [35] to solve $H(\gamma)$ exactly for $N=4 n$ or $4 n+2$ sites with periodic or antiperiodic boundary conditions, respectively. Low-energy excitations are accessible up to $N=16$, and the full spectrum to $N=10$. At constant $U$ and $t$, the condition $E_{m}(V)=E_{\sigma}(V)$ gives $V_{s}(N)$ while $E_{\sigma}(V)=E_{J}(V)$ gives $V_{c}(N)$. We also
TABLE I: Boundaries $V_{s}$ and $V_{c}$ of the BOW phase of $H(\gamma)$, Eq. 11 with $N$ sites, $t=1, U=8, \gamma=0.2$, and periodic (antiperiodic) boundary conditions for $N=4 n(4 n+2)$ based on excitation thresholds $E_{m}, E_{\sigma}, E_{J}$.

\begin{tabular}{c|c|c|c}
\hline$N$ & $V_{s}\left(E_{\sigma}=E_{m}\right)$ & $V_{1}\left(E_{\sigma}=0\right)$ & $V_{c}\left(E_{\sigma}=E_{J}\right)$ \\
\hline 8 & 4.834 & 5.105 & 5.199 \\
10 & 4.908 & 5.139 & 5.201 \\
12 & 4.908 & 5.139 & 5.201 \\
14 & 4.932 & 5.149 & 5.202 \\
16 & 4.952 & 5.157 & 5.203 \\
\hline
\end{tabular}

TABLE II: Magnetic gap $E_{m}(V)$ to the lowest triplet of $H(\gamma)$, Eq. 1 with $N$ sites, $t=1, U=8, \gamma=0.2$, and periodic (antiperiodic) boundary conditions for $N=4 n(4 n+2)$.

\begin{tabular}{c|c|c|c}
\hline$N$ & $E_{m}\left(V_{1}\right)$ & $E_{m}\left(V_{c}\right)$ & $E_{m}\left(V_{c}+0.3\right)$ \\
\hline 8 & 0.325 & 0.496 & 1.247 \\
10 & 0.268 & 0.451 & 1.293 \\
12 & 0.263 & 0.413 & 1.340 \\
14 & 0.257 & 0.384 & 1.378 \\
16 & 0.242 & 0.364 & 1.403 \\
\hline
\end{tabular}

define $V_{1}(N)$ where $E_{\sigma}(V)=0$ and the degenerate gs in the BOW phase can be explicitly constructed as linear combinations of $\sigma= \pm 1$ functions [30]. Table I lists $V \mathrm{~s}$ in units of $t$ for $\gamma=0.2\left(\alpha_{M}=1.6\right)$ and $U=8 t$ in Eq. 1] up to $N=16$. The $V$ s cluster as expected about $U / \alpha_{M}=5.0$. Their weak $N$ dependence makes it possible to extrapolate to the extended system as discussed [36, 37] in connection with a frustrated spin chain. We have computed $V_{s}(N), V_{1}(N)$ and $V_{c}(N)$ of all three models (EHM, EEHM, PCM) as functions of $U<U^{*}$ and have previously reported [30] EHM values at $U=4 t$.

The magnetic gap $E_{m}$ dominates $\chi(T)$ as $T \rightarrow 0$. It opens [26, 33] slowly at $V_{s}$, remains small at $V_{1}$ and grows rapidly on crossing the CDW boundary at $V_{c}$. The size dependence of $E_{m}$ in Table II is for the EEHM at $U=8 t$. Decreasing $E_{m}(N)$ is found in spin or Hubbard chains with $E_{m}=0$ in the extended system. Instead, $E_{m}$ increases with $N$ in all three models when $V$ slightly exceeds $V_{c}(N)$. A density matrix renormalization group (DMRG) calculation 33. for the EHM at $U=4 t$ shows increasing $E_{m}(N, V)$ for $N>30$ at $V=V_{c}(N)$. Hence $E_{m}$ of the extended system may exceed the $N=8$ gaps that we use below for $V \approx V_{c}$ in the BOW phase for $V>V_{c}$ in the CDW phase.

We compute the full spectrum of $H(\gamma)$ for $N=8(10)$ sites with periodic (antiperiodic) boundary conditions [30]. Standard methods give the partition function $Q$ and molar spin susceptibility $\chi(T)$. Fig. 2 shows $\chi(x)$ as a function of reduced temperature $x=k_{B} T / t$, where $k_{B}$ is the Boltzmann constant. Since $g\left(\mathrm{TCNQ}^{-}\right) \approx 2.00236$, the free-electron value, $\chi(x)$ is directly related to Avogadro's number $N_{A}$ and the Bohr magneton $\mu_{B}$. Jüttner et al. [38] obtained quantitative $\chi(x)$ for the Hubbard 


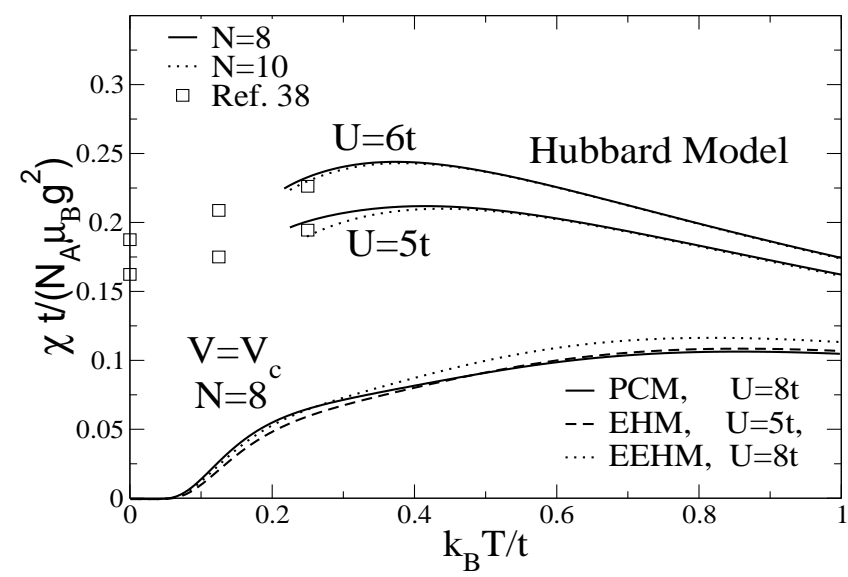

FIG. 2: Molar magnetic susceptibility $\chi$ of Hubbard-type models, Eq. 1 with finite $N$. The $U=5 t$ and $6 t, V=0$ curves for $N=8$ and 10 match the extended results (open symbols) from ref. 38] for $k_{B} T>0.3 t$. The other curves have $V=V_{c}$. The EHM has $U=5 t$, the EEHM has $U=8 t$ and $\chi=0.2$ in Eq. 1 and the PCM has $U=8 t$ and Coulomb interactions $V_{n}=V / n$.

model with $V=0$ in Eq. 1 and finite $\chi(0)$; their results for $U=5 t$ and $6 t$ are shown by open symbols in Fig. 2. The lines are exact $N=8$ and 10 results that for $x>0.3$ coincide with the extended chain within our ability to read graphs. The other $\chi(x)$ curves in Fig. 2 are for $N=8$ with periodic boundary conditions and $V=V_{c}$. We find similar $\chi(x)$ for the EHM with $U=5 t$, the EEHM with $U=8 t, \gamma=0.2$ and the PCM with $U=8 t$. Small $V$ is conveniently approximated as a Hubbard model with an effective $U_{e}=U-V$. This rationalizes reduced $\chi(x)$ with increasing $V$, but not the qualitative change of $\chi(0)=0$ due to finite $E_{m}$ in the BOW or CDW phase. Large $t \approx 1000 K$ and limited thermal stability of ion-radical oganic solids limits $\chi(x)$ to $x<0.5$.

\section{MAGNETIC SUSCEPTIBILITY}

In this Section, we model $\chi(T)$ data in Fig. 1 using regular stacks for $\mathrm{Rb}(\mathrm{II})$ and for $\mathrm{K}$ and $\mathrm{Na}$ at $T>T_{d}$. The first terms of Eq. 1 for dimerized stacks at $T<T_{d}$ has transfer integrals

$$
t_{p}=-\left(1+(-1)^{p} \delta\right)
$$

along the stack. We did not change $V$ in dimerized stacks. Since all three salts have $\mathrm{TCNQ}^{-}$stacks, similar $U$ is expected on physical grounds, and we have sought similar $U<U^{*}$ without strictly enforcing the constraint.

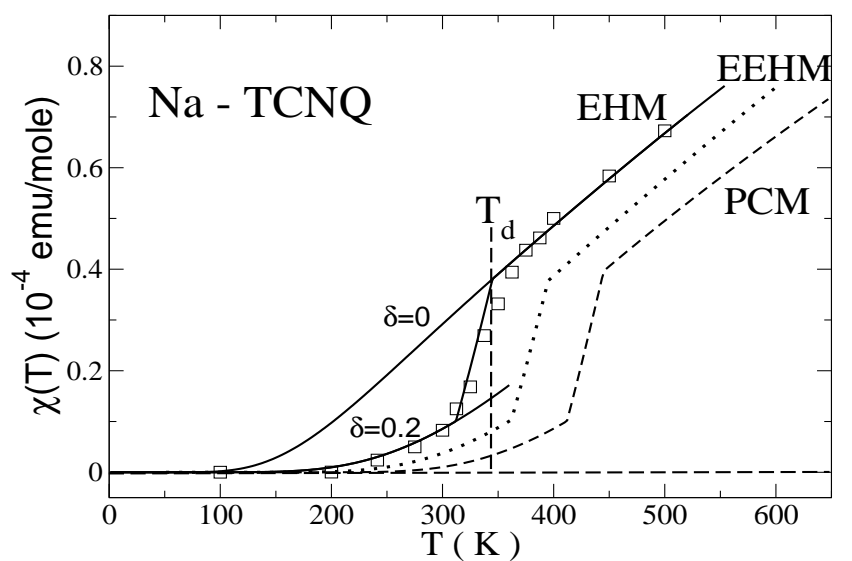

FIG. 3: Three $\chi_{M}(T)$ fits shifted by $50 \mathrm{~K}$ for clarity of the Na-TCNQ data (open symbols) in Fig. 11 for Hubbard-type models in Eq. 1 with $N=8$ and parameters in Table III. The stacks are regular $(\delta=0)$ for $T>T_{d}$, dimerized $(\delta=0.20)$ at low $T$ and interpolated using Eq. 3 in between.

It soon became apparent that $\chi(T)$ for $T>T_{d}$ requires $V>V_{c}$. We studied the PCM with $V_{n}=V / n$ and EEHM with $\gamma=0.2$ in Eq. 1 in addition to the EHM $(\gamma=0)$ in part to search for a fit with $V \leq V_{c}$ and in part to probe the dependence of $t$ and $U$ on the model. The following $\chi(T)$ calculations are all for $N=8$ sites with periodic boundary conditions in Eq. 1. The experimental data in Fig. 1 are now shown as open symbols.

We start with $\chi(T)$ for Na-TCNQ in Fig. 3 and obtain good fits for $T>T_{d}$ for the EHM with $U / t=4, t=$ $0.097 \mathrm{eV}$ and $V / t=V_{c}+0.19$. The $\chi(T)$ results for the EEHM and PCM are displaced by 50 and $100 \mathrm{~K}$, respectively, for clarity. They are equally good for the $t, U$ and $V$ parameters listed in Table III. All three models return $t \approx 0.10 \mathrm{eV}$ and $V$ slightly larger than $V_{c}$. Good $\chi(T)$ fits to $T=310 \mathrm{~K}$ in the dimerized phase are shown in Fig. [3 with $\delta=0.20$ in Eq. 2 and the same $t$, $U$ and V. Konno and Saito 13 . followed the temperature dependence of the Na-TCNQ crystal structure and found a coexistence region. The regular phase for $T>T_{d}=$ $345 \mathrm{~K}$ appears already at $T=332 \mathrm{~K}$ and grows at the expense of the dimerized phase that disappears at $T_{d}$. The $\chi(T)$ fits in Fig. 3 between $T_{1}=310 \mathrm{~K}$ and $T_{d}$ are linear interpolations according to

$$
\chi(T)=\frac{T-T_{1}}{T_{d}-T_{1}} \chi(T, \delta=0)+\frac{T_{d}-T}{T_{d}-T_{1}} \chi(T, \delta=0.2)
$$

The coexistence region is $10 \mathrm{~K}$ wider in the fit. Terauchi [9] studied the intensity of selected superlattice 
TABLE III: Parameters for the spin susceptibility of $\mathrm{Na}, \mathrm{K}$ and Rb-TCNQ(II) in Figs. 3/45

\begin{tabular}{c|c|c|c|c}
\hline Salt & Model & $t(e V)$ & $U(e V)$ & $V(e V)$ \\
& & & & \\
\hline \multirow{2}{*}{$\mathrm{Na}$} & EHM & 0.0956 & 0.383 & 0.214 \\
& EEHM & 0.0969 & 0.630 & 0.434 \\
& PCM & 0.0965 & 0.627 & 0.492 \\
\hline \multirow{2}{*}{$\mathrm{K}$} & EHM & 0.0780 & 0.370 & 0.211 \\
& EEHM & 0.0801 & 0.601 & 0.406 \\
& PCM & 0.0758 & 0.569 & 0.440 \\
\hline \multirow{2}{*}{$\mathrm{Rb}$} & EHM & 0.0745 & 0.373 & 0.199 \\
& EEHM & 0.0767 & 0.614 & 0.399 \\
& PCM & 0.0707 & 0.601 & 0.440 \\
\hline
\end{tabular}

reflections for $T<T_{d}$ in both $\mathrm{Na}$ and K-TCNQ. The intensity is proportional to $\delta(T)^{2}$ and decreases linearly as $T_{d}-T$ near $T_{d}$. The susceptibility between $320-345$ $\mathrm{K}$ can also be modeled as variable $\delta(T)$.

Fig. 4 shows $\chi(T)$ fits for K-TCNQ, again displaced by $50 \mathrm{~K}$ for clarity and again in the CDW phase with $V>V_{c}$ for $T>T_{d}=398 \mathrm{~K}$. The K-TCNQ parameters $t, U$ and $V$ are in Table III. The same parameters and $\delta=0.40$ agree with experiment up to $350 \mathrm{~K}$. There is no evidence of coexisting phases. The intensity of superlattice reflections decreases over an $80 \mathrm{~K}$ interval and changes discontinuously from $\delta / 2$ to 0 at the transition [9]. The solid points are calculated $\chi\left(T_{d}, \delta / 2\right)$. Agreement at $\chi\left(T_{d}\right)$ indicates that $\chi(T)$ between $350 \mathrm{~K}$ and $T_{d}$ can be fit with variable $\delta(T)$ in these models. Smaller $t(K) \approx 0.08 \mathrm{eV}$ is consistent with larger $\mathrm{R}$ in K-TCNQ.

Figure 5 shows $\chi(T)$ fits for Rb-TCNQ(II) for the three models displaced by $50 \mathrm{~K}$. We took $V=V_{c}(8)$ at the upper limit of the BOW phase and set $E_{\sigma}=0$. The $\delta=0$ fit for regular stacks is markedly improved by slightly increasing $E_{m}$ beyond $E_{m}(8) / t$ in Table II by $0.07 t$ for the EHM and by $0.10 t$ for PCM and EEHM. Finite-size effects are critical in view of other evidence [17, 30] for broken $\mathrm{C}_{\mathrm{i}}$ symmetry in Rb-TCNQ(II), which implies $V \leq V_{c}$. By contrast, finite-size effects for the $\mathrm{Na}$ or $\mathrm{K}$ salts are absorbed in $V>V_{c}$. Good $\delta=0$ fits are obtained down to $T \approx 250 \mathrm{~K}$ with the $t$ and $U$ parameters in Table III. The esr intensity in Fig. 11 has a pronounced knee around $T_{k n} \approx 220 \mathrm{~K}$. The knee is less prominent in the static susceptibility. Dimerization is ruled out by the $100 \mathrm{~K}$ structure, which has regular stacks and the $300 \mathrm{~K}$ space group [17].

An adiabatic (Born-Oppenheimer) approximation for the lattice is typically invoked to model the Peierls [39] or spin-Peierls [12, 40] instability of 1D systems, although quantum fluctuations [41] are important for small $\delta(0)$ at $T=0$. The BOW phase has finite $\delta(0)$ for linear electron-phonon (e-ph) coupling $\alpha$ to a harmonic lattice

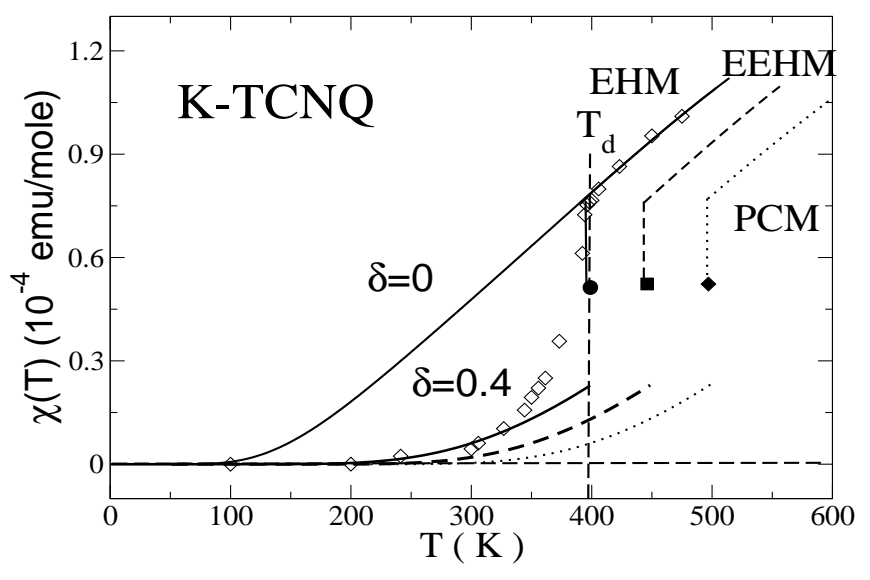

FIG. 4: Three $\chi_{M}(T)$ fits shifted by $50 \mathrm{~K}$ for clarity of the K-TCNQ data (open symbols) in Fig. 1 for Hubbard-type models in Eq. 1 with $N=8$ and parameters in Table III. The stacks are regular $(\delta=0)$ for $T>T_{d}$, dimerized $(\delta=0.40)$ at low $T$ and jump to $\delta / 2$ at $T_{d}$.

[30], where $\alpha=(d t / d u)_{0}$ is the first term of the Taylor expansion of $t(R+u)$. The electronic gs energy per site in units of $t$ has a cusp [30]

$$
\epsilon_{0}(\delta)-e_{0}(0)=-B(V)|\delta|+O\left(\delta^{2}\right)
$$

where $B(V)$ is the order parameter of the BOW phase. $B(V) \approx 0.4$ is the bond-order difference at $V=V_{1}(U)$ in Table I for all three models for the $U$ s in Table III. For comparison, a half-filled band of free electrons with $\delta= \pm 0.1$ has comparable $B(\delta)$ for partial double and single bonds.

The BOW phase has long-range order that cannot persist for $T>0$ in $1 \mathrm{D}$ systems. As discussed by $\mathrm{Su}$, Schrieffer and Heeger [39] for free electrons, the extended system at low $T$ has regions with reversed $\delta(0)$ that are separated by topological solitons whose width $2 \xi$ goes as $1 / \delta(0)$. Spin solitons are also found numerically in the BOW phase [30] of the EHM or in the magnetic properties of organic ion-radical salts [42].

We consider dimerization fluctuations in the BOW phase. This regime has equal densities $\rho(T)$ of spin solitons and dimerized segments with successively $\pm \delta(0)$ in Eq. 4. We approximate each $S=1 / 2$ soliton by a regular region of $2 \xi$ sites in an otherwise dimerized stack. Since $E_{m}$ is not degenerate, $E_{m}(V, \delta(0)) / t$ initially increases as $B(V)|\delta(0)| N$ due to the cusp in Eq. 4, as found directly [30] up to $N=16$ at $V=V_{1}(N)$ where $E_{\sigma}=0$. Such size dependence cannot go on indefinitely. It suffices for our purposes to note that $\delta(0)<0.10$ 


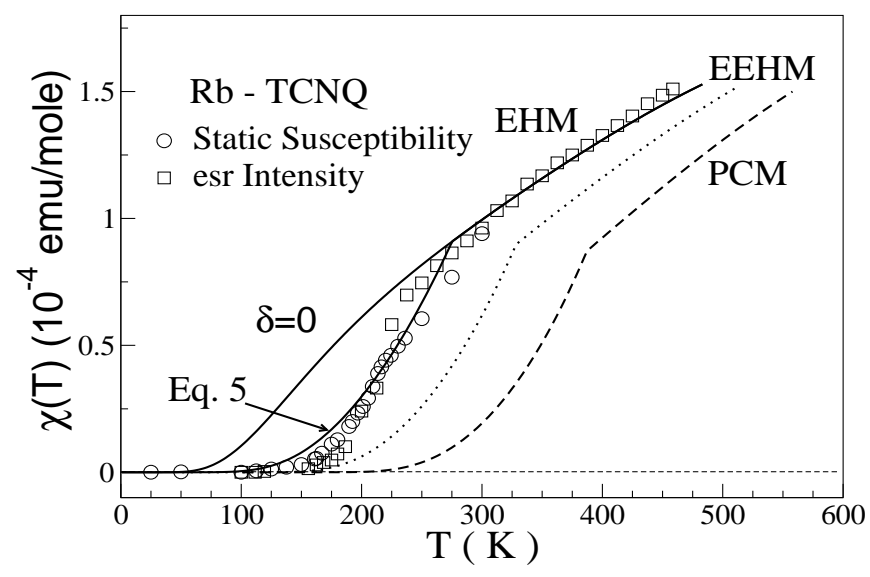

FIG. 5: Three $\chi(T)$ fits shifted by $50 \mathrm{~K}$ for clarity of the RbTCNQ(II) data (open symbols) in Fig. 1 for Hubbard-type models in Eq. 1 with $N=8$ and parameters in Table III The stacks are regular $(\delta=0)$. Spin solitons with width $2 \xi=60$ in Eq. [5] are used at low $T$.

generates large $E_{m}$ with negligible $\chi(T)$ at low $T$ in dimerized regions between solitons. Such regions decrease with increasing $T$ and vanish at $2 \xi \rho\left(T^{\prime}\right)=1$ when the stack is regular everywhere.

In this approximation, dimerization fluctuations reduce $\chi(T)$ for $T<T^{\prime}$. The soliton density $\rho(T)=$ $\chi(T) T / C$ follows directly from the molar Curie constant $C=N_{A} g^{2} \mu_{B}^{2}$ of noninteracting spins. The knee region in Fig. [5 up to $T$ is modeled as

$$
\chi\left(T<T^{\prime}, \xi\right)=2 \xi \rho(T) \chi(T)
$$

with $2 \xi=60$. The fit is adequate for the simple treatment of fluctuations. The choice of $2 \xi=60$ gives $T^{\prime} \approx 250 \mathrm{~K}$, somewhat higher than experiment. The same soliton width accounts for the $T$ dependence of the infrared intensity of a totally symmetric TCNQ ${ }^{-}$ vibration [30]. Such IR data is decisive evidence [43, 44] for broken $\mathrm{C}_{\mathrm{i}}$ symmetry, whether due to $B(\delta)$ in wellcharacterized K-TCNQ stacks [45] at $300 \mathrm{~K}$ or to finite $B(V)$ in a BOW phase.

It remains to reconcile dimerization fluctuations at low $T$ with the X-ray data for regular stacks at 100 $\mathrm{K}$ and thermal ellipsoids that conservatively limit [46] $\mathrm{R}_{+}-\mathrm{R}_{-}=2 u<0.04 \AA$. . To be detectable, $u$ must exceed zero-point motions. The stack at $100 \mathrm{~K}$ has small $\rho(T)$ that prevents long range order. Soliton motion modulates $\mathrm{R}$ as $\delta(0)=\alpha u / t$, and the magnitude of $\alpha / t$ determines whether $\delta(0)$ is consistent with $\mathrm{X}$-ray data.
We conclude this Section by assessing the parameters in Table [II] Three models (EHM, EEHM, PCM) with narrow BOW phases have been applied to three TCNQ salts (Na, K, Rb(II)). The CT integral $t$ of regular stacks depends on overlap, as sketched in Fig. 1, and on separation between $\mathrm{TCNQ}^{-}$planes. It is reassuring that the models return identical $t$ to better than $10 \%$ with $t(N a)>t(K)>t(R b)$ in an unconstrained fit. We sought similar $U$ in $\mathrm{TCNQ}^{-}$stacks. The $U \mathrm{~s}$ in Table III are identical within $5 \%$ for each model. The BOW/CDW boundary $V_{c}$ of the EHM with $\alpha_{M}=2$ leads to $U(E H M)<U(E E H M) \approx U(P C M) \approx 0.61 \mathrm{eV}$ that we prefer on the basis of $\alpha_{M} \approx 1.5$. The $\mathrm{Na}$ and $\mathrm{K}$ salts are in the CDW phase with $V>V_{c}$ while $\chi(T)$ of RbTCNQ(II) is consistent with a BOW phase with $V$ close to $V_{c}$. The $\chi(T)$ fit of Na leads to $\delta=0.2$ up to $T \approx 310$ $\mathrm{K}$ and the interpolation in Eq. 3 for coexisting phases up to $T_{d}$. The K-TCNQ fit has larger $\delta=0.4$ at low $T$. The knee region of Rb-TCNQ(II) is fit by Eq. 5 with $2 \xi=60$, the soliton width used previously [30] for IR data.

\section{MODEL PARAMETERS}

The parameters in Table $\llbracket$ are for three models with BOW and CDW phases. They are internally consistent, but considerably smaller than expected from optical data. Typical values [1-4] are $t \approx 0.1-0.3 \mathrm{eV}$ and $U \approx$ $1 \mathrm{eV}$ in Hubbard models or larger $U_{e}=U-V \approx 1 \mathrm{eV}$ in the EHM. Such parameters rationalize a CT transition around $1 \mathrm{eV}$ polarized along the stack and magnetic excitations at lower energy $4 t^{2} / U_{e} \approx 0.1 \mathrm{eV}$. We return in the Discussion to model parameters. Here we report direct evaluation of $U, V$ and $t$ for individual or adjacent $\mathrm{TCNQ}^{-}$. The results are based on density functional theory (B3LYP) with the $6-311^{* *} \mathrm{G}(\mathrm{p}, \mathrm{d})$ basis in the Gaussian 03 package [47]. An eclipsed (TCNQ) $)_{2}^{-2}$ dimer at $\mathrm{R}=3.2$ or $3.4 \AA$ is correctly found to have singlet gs, while smaller basis sets [48] yield a triplet gs. Smaller basis sets are adequate for model parameters, however, as discussed [49] for $t$.

The disproportionation reaction $2 A^{-} \rightarrow A^{2-}+A$ relates $U$ to the gs energies $E_{0}\left(A^{-}\right), E_{0}(A)$ and $E_{0}\left(A^{2-}\right)$. The optimized $\mathrm{TCNQ}^{-}$structure leads to $U$ (vertical $)=$ $4.413 \mathrm{eV}$. Optimization of TCNQ and $\mathrm{TCNQ}^{2-}$ returns $U($ adiabatic $)=4.192 \mathrm{eV}$. The relaxation energy of 0.22 $\mathrm{eV}$ for electron transfer is in excellent agreement with $0.1 \mathrm{eV}$ per $\mathrm{TCNQ}^{-}$deduced [43] from Raman and IR spectra. The interaction $V$ depends on adjacent $\mathrm{TCNQ}^{-}$ and can be estimated several ways: (1) electrostatic repulsion between the atomic charges $q_{i}$ of the two ions; (2) repulsion between $q_{i}$ obtained in a dimer calculation; (3) energy difference ${ }^{3} E_{0}-2 E_{0}\left(A^{-}\right)$between the triplet gs of the dimer, which precludes the formation of a $\pi-\pi$ bond, and two radical ions. The same values are obtained [48] to better than $5 \%$, and $V \mathrm{~s}$ in Table IV are based on the triplet. The listed $V(N a)$ and $V(K)$ are for eclipsed 
TABLE IV: Calculated model parameters for adjacent $\mathrm{TCNQ}^{-}$

\begin{tabular}{c|c|c|c}
\hline Parameter & Na-TCNQ & K-TCNQ & Rb-TCNQ(II) \\
\hline$V(\mathrm{eV})$ & 2.713 & 2.671 & 2.594 \\
$t(\mathrm{eV})$ & 0.345 & 0.299 & 0.182 \\
$t_{1} / t_{2}(\mathrm{eV})$ & $0.299 / 0.468$ & $0.254 / 0.444$ & - \\
$t_{1} / t_{2}(\mathrm{eV})$ & $0.266 / 0.451$ & $0.265 / 0.429$ & - \\
$\alpha(\mathrm{eV} / \AA)$ & 0.59 & 0.55 & 0.34 \\
\hline
\end{tabular}

$(\mathrm{TCNQ})_{2}^{2-}$ with $\mathrm{R}=3.385$ and $3.479 \AA$, respectively. The regular Rb-TCNQ(II) stack has $\mathrm{R}=3.241 \AA$ and a $2.0 \AA$ displacement along the long axis shown in Fig. 1.

The ratio $U(a d) / V \approx 1.5$ is comparable to $\alpha_{M}=1.6$ for $\gamma=0.2 \mathrm{in} \mathrm{Eq.} 1$ or to $\alpha_{M}(8)=17 / 12$ for an 8-site PCM. The 1D stack is close to the CDW boundary of the EEHM or PCM. The magnitude of $U$ is strongly reduced in the solid state by electronic polarization $P \approx 1 \mathrm{eV}$ per charge [50]. Since $P$ is approximately quadratic in charge, we have $P\left(A^{2-}\right)-2 P\left(A^{-}\right) \approx 2 \mathrm{eV}$. Electronic polarization of adjacent $A^{-}$reduces $V$ by a smaller amount.

The $t$ s in Table [V] are for the $300 \mathrm{~K}$ structure of $\mathrm{Rb}$ and the $T>T_{d}$ structures of $\mathrm{Na}$ and $\mathrm{K}$. We find $t(N a)>t(K)>t(\mathrm{Rb})$ as expected but for calculated $t$ s that exceed the magnetic parameters in Table III by a factor of 2.5 for $\mathrm{Rb}$ and 3.5 for $\mathrm{Na}$ or $\mathrm{K}$. The reason for such large reduction is not understood. There are two dimerized stacks [5] in Na or K-TCNQ at $300 \mathrm{~K}$. Table IV lists the calculated $t_{1}, t_{2}$ and the larger, smaller separation $\mathrm{R}_{+}, R_{-}$. We obtain $\delta(N a)=\left(t_{2}-t_{1}\right) /\left(t_{2}+t_{1}\right)=0.22$ or 0.26 , somewhat larger than $\delta=0.20$ at low $T$ in Fig. 3. The corresponding $\delta(K)$ are 0.27 and 0.25 , smaller than $\delta=0.40$ in Fig. 4. But the $\mathrm{K}$ salt has substantially larger $\left(t_{1}+t_{2}\right) / 2=1.17 t$ in the dimerized phase that leads to an equally good $\chi(T)$ for $\delta=0.30$ when the mean value of the transfer integral is used. Overall, the calculated and fitted $\delta$ are reasonably consistent.

The two-point derivative $d t / d \mathrm{R}=\alpha$ is an estimate for the e-ph coupling constant. The two stacks of $\mathrm{Na}$ or K-TCNQ at $T<T_{d}$ have almost the same $\alpha$, whose average value is reported in Table [V] The 300 and $100 \mathrm{~K}$ structures of Rb-TCNQ(II) return a smaller $\alpha \approx 0.34 \mathrm{eV} / \AA$. The structural constraint of $2 u<0.04 \AA$ discussed above leads to $\delta(0)<\alpha u / t=0.036$, an estimate that is independent of reduced $t$ in the crystal. Dimerization fluctuations of such small amplitude would be difficult to detect.

Direct evaluation of $t$ has been discussed before [49, 51]. Eclipsed $\mathrm{TCNQ}^{-}$gives the largest $t(\mathrm{R}, 0)$ that decreases with increasing separation R. Displacing the ion by $\mathrm{L}$ along the long axis leads to tilted stacks in Fig. 1. The nodes of the singly occupied orbital of $\mathrm{TCNQ}^{-}$ generate $t(\mathrm{R}, \mathrm{L})=0$ at $\mathrm{L}=1.3 \AA$ and to secondary maxima at other $\mathrm{L}$ [49, 52]. The first maximum at $\mathrm{L}$ $=2.1 \AA$ is close to the Rb-TCNQ(II) or TTF-TCNQ structures. A series of substituted perylenes illustrates wider variations of $t$ with displacements along both the long and short molecular axes [51].

We consider next parameters derived from nonmagnetic data. Simple TCNQ salts have a broad CT absorption $\hbar \omega_{C T} \approx 1 \mathrm{eV}$ polarized along the stack. The optical conductivity of K-TCNQ has a shoulder at higher energy that has been variously associated with dimerization [53], with a band edge [54] or with a local excited state [45] of $\mathrm{TCNQ}^{-}$. Meneghetti [55] modeled K-TCNQ with special attention to totally symmetric mid-IR modes that are coupled in dimerized stacks to the CT absorption. Polarized spectra yield the coupling constants $g_{n}$. Meneghetti [55] used an EHM with $N=4$ sites, periodic boundary conditions, and adjustable $t_{1}$, $t_{2}$ and $V_{1}, V_{2}$ at $T<T_{d}$. Comparison with experiment also entails lifetime or broadening parameters. Nearly quantitative fits are shown in Fig. 8 of ref. [55] for the optical conductivity at $300 \mathrm{~K}$ with coupled mid-IR modes and in Fig. 7 for polarized spectra at 27, 300 and $413 \mathrm{~K}$.

The EHM parameters of ref. [55] for a regular KTCNQ stack are $t=0.19 \mathrm{eV}, U=1.20 \mathrm{eV}$ and $V=0.02$ $\mathrm{eV}$. Neglecting $V$ for a moment, we have a Hubbard model with $\chi(0) \approx 1.0 \times 10^{-4} \mathrm{emu} /$ mole. Including $V=0.02 \mathrm{eV}$ in a $N=8$ calculation leads to $\chi(T)$ with a broad maximum at $1.6 \times 10^{-4} \mathrm{emu} /$ mole at $T_{\max } \approx 800$ $\mathrm{K}$, consistent with the magnitude of regular stacks in Fig. 1. The observed $\chi(T)$ slope for $T>T_{d}$ is much steeper, however, and finite $\chi(0)$ is not consistent with Rb-TCNQ(II). We note that $V=0.02 \mathrm{eV}$ is a finite-size effect since $N=4$ confines an e-h excitation to be close together. The CT absorption shifts to lower energy with increasing $\mathrm{N}$ and optical spectra of longer regular stacks return different parameters. The $300 \mathrm{~K}$ parameters of ref. [55] are again $U=1.20 \mathrm{eV}$ and alternating $t_{1}=0.10$, $t_{2}=0.37 \mathrm{eV}($ or $\delta=0.27 / 0.47=0.574)$ and $V_{1}=0.28$, $V_{2}=0.31 \mathrm{eV}$. The strong CT absorption at $N=4$ hardly shifts to the red at $N=8$ or 12 . But $\delta=0.574$ opens a large $E_{m}$. The calculated $\chi(T)$ for $N=8$ with these parameters is very small $\left(<10^{-6} \mathrm{emu} /\right.$ mole $)$ up to $500 \mathrm{~K}$, completely incompatible with the magnetic data in Fig. 1.

Quantitative treatment of e-mv coupling in dimerized stacks such as K-TCNQ is based on linear response theory and force fields for molecular vibrations [56, 57]. The coupling constants $g_{n}$ depend on just one electronic parameter, the zero-frequency optical conductivity. While the CT band is of central importance, its precise modeling is not. Dimerized stacks with broken $\mathrm{C}_{\mathrm{i}}$ symmetry are required for coupling to mid-IR modes. Coupling to the same mid-IR modes in Rb-TCNQ(II) in regular stacks is strong evidence for a BOW phase 
with broken $\mathrm{C}_{\mathrm{i}}$ symmetry. The same modes appear [58] with slightly higher intensity in powder spectra of Rb-TCNQ(I), which is dimerized [15] at $300 \mathrm{~K}$. The $T$ dependence of the intensities $I_{\mathrm{IR}}(T)$ of coupled modes is characteristic of a BOW phase, and spin solitons with $2 \xi=60$ account [30] for $I_{\mathrm{IR}}(T)$.

The optical spectrum in the narrow BOW phase is dominated by $t$ due to competition between the larger $U$ and $V$ terms. The CT absorption peak is around $3 t$ for a regular stack of rigid molecules 34] and shifts to higher energy by $U$ (vert) $-U($ ad $)=0.22$ eV. Dimerization also shifts $\hbar \omega_{C T}$ to higher energy. Preliminary modeling with all eigenstates of $N=8$ or 10 indicates that the $t \mathrm{~s}$ in Table $\amalg$ III have to be doubled for optical spectra and that $\delta \approx 0.3$ produces small blue shifts without a shoulder on the high-energy side. Larger $t$ s have been assumed all along for optical spectra.

\section{DISCUSSION}

We have modeled the molar spin susceptibility $\chi(T)$ of alkali-TCNQ salts in Fig. 1 that were previously beyond quantitative treatment. We have not treated the phase transitions of $\mathrm{Na}$ or K-TCNQ, but relied on crystal data for $T_{d}$ and coexisting phases or diffuse scattering. We found consistent parameters in Table Ifor 1D Hubbard models with point charges or second-neighbor $V$ that reduced the Madelung constant to $\alpha_{M} \approx 1.5$. The $\chi(T)$ fits in Fig. 3/45 have $t, U, V$ parameters in Table III that are about half as large as parameters from optical data.

It should perhaps be no surprise that quantitative analysis of magnetic and optical data within the same model leads to different parameters. Hubbard models make the zero-differential-overlap (ZDO) approximation of Hückel theory for conjugated molecules or of tight-binding theory in solids. The PCM with $V_{n}=V / n$ is a special case of the Pariser-Parr-Pople (PPP) model [59, 60]. Salem [60] has summarized the merits and limitations of ZDO, which does not concern us here. But his discussion of $t$, the Hückel $\beta$ parameter, bears directly on different magnetic and optical parameters. Systematic variations are illustrated by many conjugated hydrocarbons with $\mathrm{sp}^{2}$ hybridized $\mathrm{C}$ atoms. Hückel theory provided a convenient approach to analyze variations prior to modern digital computers. Thermochemical data were successfully fit with a $\beta_{t h}$ that is roughly half of $\beta_{o p}$ inferred from optical spectra 60]. The correlated PPP model with $\beta_{o p}$ is defined by the geometry of planar conjugated molecules and has considerable predictive power [57, 61], including two-photon spectra and nonlinear optical properties. More recently, INDO (intermediate neglect of differential overlap) and its spectroscopic version INDO/S have different $\beta$ parameters [62].

Instead of closely related hydrocarbons, Hubbard models are used to study electron-electron correlation solids in general. Quantitative application is rare and so are homologous series. Moreover, magnetic and optical or other properties are typically modeled separately and a single half-filled Hubbard band is rarely thought to be quantitative. $\mathrm{Na}, \mathrm{K}$ and $\mathrm{Rb}-\mathrm{TCNQ}$ (II) are closely related quasi-1D systems that nevertheless crystallize in different space groups.

At least qualitatively, differences between magnetic and optical parameters may be rationalized in terms of relaxed states in thermal equilibrium and electronic excitations that are fast compared to atomic or molecular motions. Equilibrium states that contribute to $\chi(T)$ are fully relaxed with respect to both molecular and lattice modes, and relaxed states have reduced excitation energies. Hubbard or other approaches to electronic excitations start with vertical $0-0$ excitations. Electronic polarization reduces $U$ and $V$ significantly in the solid state, but this fast process is fully included in model parameters for optical spectra. The Holstein model [63] illustrates reduced $t$ due to linear coupling to a molecular vibration. Lattice phonons are considered in 1D for selected modes such as the Peierls mode but complete 3D relaxation is prohibitively difficult. Yet such relaxation is the most likely explanation for small parameters derived from $\chi(T)$ data. Quantitative modeling of the spin susceptibility clearly points to different magnetic and optical parameters for $\mathrm{Na}, \mathrm{K}$ and Rb-TCNQ(II). The magnetism also indicates the Hubbard-type models for the $\mathrm{Na}$ and $\mathrm{K}$ salts at $T>T_{d}$ are in the CDW phase while the $\mathrm{Rb}(\mathrm{II})$ salts is in the BOW phase close to the CDW boundary.

\section{Acknowledgements.}

ZGS thanks A. Girlando for access to unpublished IR spectra, A. Painelli for stimulating discussions about 1D models and their instabilities, and R. Pascal, Jr., for analysis of X-ray data. We gratefully acknowledge support for work by the National Science Foundation under the MRSEC program (DMR-0819860).
[1] Z.G. Soos, Ann. Rev. Phys. Chem. 25, 121 (1974).

[2] Z.G. Soos and D.J. Klein, in: N.B. Hannay (Ed.) Treatise on Solid-State Chemistry, (Plenum, New York, 1976, Vol. III) p. 689; Z.G. Soos and S.R. Bondeson, in Extended
Linear Chain Compounds, J.S. Miller, Ed. (Plenum, New York, 1983, Vol. 3) p. 193.

[3] J.B. Torrance, Acct. Chem. Res. 12, 79 (1979).

[4] D. Jerome, Chem. Rev. 104, 5565 (2004); D. Jerome and 
H.J. Schultz, Adv. Phys. 51, 293 (2002).

[5] H. Endres, in Extended Linear Chain Compounds, J.S. Miller, Ed. (Plenum, New York, 1983, Vol. 3) p. 263.

[6] P.L. Nordio, Z.G. Soos, and H.M. McConnell, Ann. Rev. Phys. Chem.17, 237 (1966).

[7] K. Ikegami, K. Ono, J. Togo, T. Wakabayashi, Y. Ishige, H. Matsuzaki, H. Kishida and H. Okamoto, Phys. Rev. B. 76, 085106 (2007).

[8] H. Uemura, H. Matsuzaki, Y. Takahashi, T. Hasegawa and H. Okamoto, Physica B 405 S357 (2010).

[9] H. Terauchi, Phys. Rev. B 17, 2446 (1978).

[10] J.B. Torrance, Ann. N.Y. Acad. Science 313, 210 (1978).

[11] Y. Lepine, C. Tannous and A. Caille, Phys. Rev. B 20, 3753 (1979); Y. Lepine, Phys. Rev. B 28, 2659 (1983); G. Beni and P. Pincus, J. Chem Phys.57, 3531 (1972).

[12] J.W. Bray, L.V. Interrante, I.S. Jacobs and J.C. Bonner, in Extended Linear Chain Compounds, J.S. Miller, Ed. (Plenum, New York, 1983, Vol. 3) p. 353.

[13] M. Konno, T. Ishii and Y. Saito, Acta Cryst. B 33, 763 (1977).

[14] M. Konno and Y. Saito, Acta Cryst. B 31, 2007 (1975).

[15] A. Hoekstra, T. Spoelder and A. Vos, Acta Cryst. B 28, 14 (1972).

[16] H. Kobayashi, Bull. Chem. Soc. Jpn. 54, 3669 (1981).

[17] T.M. McQueen, D.M. Ho, C. Jimenez Cahua, R.J. Cava, R.A. Pascal, Jr., and Z.G. Soos, Chem. Phys. Lett. 475, 44 (2009).

[18] J.G. Vegter and J. Kommandeur, Mol. Cryst. Liq. Cryst. 30, 11 (1975).

[19] R. Bozio and C. Pecile, J. Chem. Phys.67, 3864 (1977).

[20] R. B. Griffiths, Phys. Rev. 133, A768 (1964).

[21] M. Takahashi, Prog. Theor. Phys.42, 1098 (1969);43, 1619 (1970).

[22] J.E. Hirsch, Phys. Rev. Lett.53, 2327 (1984); Phys. Rev. B 31, 6022 (1985).

[23] A. Painelli and A. Girlando, J. Chem. Phys. 84, 5655 (1986),87, 1705 (1987)

[24] Z.G. Soos, S. Kuwajima and R.H. Harding, J. Chem. Phys. 85, 601 (1986)

[25] Z.G. Soos, S.A. Bewick, A. Painelli and A. Girlando, Synth. Met. 155, 3577 (2005).

[26] M. Nakamura, Phys. Rev. B 61, 16377 (2000); J. Phys. Soc. Jpn.68, 3123 (1999).

[27] P. Sengupta, A.W. Sandvik and D.K. Campbell, Phys. Rev. B 65, 155113 (2002); A. Sandvik, L. Barents and D.K. Campbell, Phys. Rev. Lett. 96, 036408 (2004).

[28] S. Ejima and S. Nishimoto, Phys. Rev. Lett. 99, 216403 (2007).

[29] Y.Z. Yang, Phys. Rev. Lett. 92, 246404 (2004); S. Glocke, A. Klumper and J. Sirker, Phys. Rev. B 76, 155121 (2007).

[30] M. Kumar and Z.G. Soos, Phys. Rev. B 82, 155144 (2010).

[31] Z.G. Soos, M. Kumar, S. Ramasesha and R.A. Pascal, Jr., Physica B 405, 5353 (2010).

[32] R.M. Metzger, in Crystal Cohesion and Conformational Energies, Topics in Current Phys. 26, R.M. Metzger, Ed. (Springer-Verlag, Berlin, 1981) p. 80.

[33] M. Kumar, S. Ramasesha and Z.G. Soos, Phys. Rev. B 79, 035102 (2009).

[34] M. Kumar, S. Ramasesha, R.A. Pascal, Jr. and Z.G. Soos, Europhys. Let. 83, 37001, (2008).

[35] Z.G. Soos and S. Ramasesha, Phys. Rev. B 29, 5410 (1984); S. Ramasesha and Z.G. Soos, in Theoretical and
Computational Chemistry, Vol. 10, D.L. Cooper, Ed. (Elsevier, Amsterdam, 2002) p. 635.

[36] K. Okamoto and K. Namura, Phys. Lett. A 169, 433 (1992).

[37] M. Kumar, S. Ramasesha and Z.G. Soos, Phys. Rev. B Phys. Rev. B 81, 054413 (2010).

[38] G. Jüttner, A. Klümper and J. Suzuki, Nuclear Physics B 522, 471 (1998)

[39] W.P. Su, J.R. Schrieffer and A.J. Heeger, Phys. Rev. B 22, 2099 (1982); A.J. Heeger, S. Kivelson, J.R. Schrieffer and W.P. Su, Rev. Mod. Phys. 60, 81 (1988).

[40] Z.G. Soos and S.A. Bewick, Chem. Phys. Lett.421, 210 (2006).

[41] W.P. Su, Solid State Commun. 42, 497 (1982).

[42] S.A. Bewick and Z.G. Soos, Chem. Phys.325, 60 (2006).

[43] R. Bozio and C. Pecile, in Spectroscopy of Advanced Materials, Adv. Spectrosc. Vol. 19, R.J.H. Clark and R.E. Hester, Eds. (Wiley, New York, 1991), p. 1.

[44] M.J. Rice, Solid State Commun. 31, 93 (1979); A. Painelli and A. Girlando, Phys. Rev. B 37, 5748 (1988); 39, 9663 (1989).

[45] D.B. Tanner, C.S. Jacobsen, A.A. Bright and A.J. Heeger, Phys. Rev. B 15, 3283 (1977).

[46] R.A. Pascal, Jr. personal communication, 2010.

[47] M.J. Frisch, et al. Gaussian 03, Revision C.02, (Gaussian, Inc. Wallingford, CT, 2003).

[48] R.H. Yu, Senior Thesis, Princeton University, 2011 (unpublished).

[49] Kertesz, t. J. Huang and M. Kertesz, Chem. Phys. Lett. 390, 110 (2004); J. Chem. Phys. 122, 234707 (2005).

[50] E.V. Tsiper and Z.G. Soos, Phys. Rev. B 64195124 (2001); Phys. Rev B 68, 085301,(2003).

[51] P.M Kazmaier and R. Hoffmann, J. Amer. Chem. Soc. 116, 9684 (1994).

[52] Q.B. Dang Ha, Senior Thesis, Princeton University, 2009 (unpublished).

[53] H. Okamoto, Y. Tokura and T. Koda, Phys. Rev. B 36, 3858 (1987).

[54] K. Yakushi, T. Kusaka and H. Kuroda, Chem. Phys. Lett. 68, 139 (1979).

[55] M. Meneghetti, Phys. Rev. B 44, 8554 (1991).

[56] A. Painelli, A. Girlando and C. Pecile, Solid State Commun. 52, 801 (1984).

[57] Z.G. Soos, D. Mukhopadhyay, A. Painelli and A. Girlando, in Handbook of Conducting Polymers, Sec. Ed., T.A. Skotheim, R. Elsenbaumer, and J.R. Reynolds, Eds.(Marcel Dekker, New York, 1998), p. 165.

[58] R. Bozio, I. Zanon, A. Giralndo and C. Pecile, J. Chem. Soc. Faraday Trans. 2, 74. 235 (1978)

[59] R. Pariser, R.G. Parr, J. Chem. Phys. 21, 767 (1953); J.A. Pople, Trans. Faraday Soc. 42, 1375 (1953).

[60] L. Salem, The Molecular Orbital Theory of Conjugated Molecules, (Benjamin, New York, 1966). Chapt.3

[61] G. Wen and Z.G. Soos, J. Chem. Phys. 108, 2486 (1998); R.G. Kepler and Z.G. Soos, Phys. Rev. B. 43, 12530 (1991); S. Ramasesha, D.S. Galvao and Z.G. Soos, J. Phys. Chem. 97, 2823 (1993).

[62] M.C. Zerner, G.H. Leow, R.F. Kirchner and U.T. Mueller-Westerhoff, J. Amer. Chem. Soc. 102, 589 (1980).

[63] T. Holstein, Ann. Phys. 8, 325 (1953); Ann. Phys. 8, 343 (1953). 